\title{
Caracterización actitudinal e intencional de la violencia juvenil exogrupal en la Comunidad de Madrid
}

\author{
María-Jesús Martín*, Barbara Scandroglio, José-Manuel Martínez y Jorge López
}

Dpto. de Psicología Social y Metodología. Universidad Autónoma de Madrid

\begin{abstract}
Resumen: Introducción. El presente artículo expone el proceso de construcción de un instrumento para medir e identificar las creencias relevantes que jóvenes integrados en grupos violentos poseen sobre la propia conducta violenta que realizan; una vez identificadas, se utilizan para explicar el desarrollo de la intención de llevar a cabo este tipo de conductas. Metodología. Se realizaron tres estudios independientes y complementarios. $\mathrm{El}$ primero $(N=216)$ identifica las creencias salientes sobre violencia. El segundo $(N=250)$ muestra el proceso de construcción del Cuestionario Piloto sobre Violencia Juvenil Exogrupal y se procede a la selección estadística de las creencias relevantes. El tercer estudio $(N=243)$ muestra el diseño definitivo del Cuestionario de Investigación de la Conducta Violenta Exogrupal y presenta el modelo de regresión realizado para explicar la intención de realizar conductas violentas. Resultados. El modelo final obtenido explica el 61.5\% de la varianza de la variable dependiente "Intención" e identifica las creencias más relevantes para explicar, tanto el desarrollo como la inhibición de la intención de realizar conductas violentas por parte de jóvenes pertenecientes a grupos. Discusión. Los resultados pueden contribuir en la fundamentación del diseño, aplicación y evaluación de intervenciones que tengan como objetivo principal la prevención o el tratamiento del problema.

Palabras clave: Violencia grupal juvenil; salud; actitudes; intención conductual; análisis de regresión; prevención.
\end{abstract}

\section{Introducción}

En España se ha constatado la existencia de grupos violentos de ideología extrema que están detrás de actos de violencia y que, pese a presentar una baja incidencia en las tasas delincuenciales, generan una especial preocupación en nuestra sociedad (Fiscalía General del Estado, 2011). La necesidad de controlar las actividades realizadas por estos grupos obligó a la Secretaria de Estado de Seguridad (Ministerio del Interior) a implantar y desarrollar un "Plan de Actuación y Coordinación Policial" que diera respuesta a este tipo de violencia. Este marco de actuación se recogió en la Instrucción 23/2005, sustituida actualmente por la Instrucción 6/2009. Los datos oficiales indican (Ministerio de Interior, 2009) que en 2008 estaban activos 192 grupos juveniles violentos y se identificaron a más de 15.000 personas relacionadas con ellos, de las que fueron detenidas 2.593. Si bien actualmente se observa una disminución de los delitos cometidos por menores, se ha constatado la existencia de bandas juveniles como los Baby Brothers, Netas o Bloods, entre otros, aunque lo más habitual es la realización de conductas violentas por parte de grupos de diferente ideología y poco organizados (Fiscalía General del Estado, 2011).

* Dirección para correspondencia [Correspondence address]

María Jesús Martín. Dpto. de Psicología Social y Metodología. Universidad Autónoma de Madrid. Ciudad Universitaria Cantoblanco. 28049 Madrid (España).

E-mail: mariajesus.martin@uam.es
Title: Attitudinal and intentional characterisation of juvenile group violence in the Madrid Area.

Abstract: Purpose. This article presents the process followed for the construction of an instrument to measure and identify the relevant beliefs owned by the youth belonging to violent groups about their own violent behaviour. Once identified, they are used as an explanation of the process of the intention to carry out this behaviour. Methodology. For that purpose, we conducted three independent but complementary. The first of them $(N=216)$ identifies the salient beliefs about violence. The second $(N=250)$ shows the process of construction of the Cuestionario Piloto sobre Violencia Juvenil Exogrupal and proceeds to the statistical selection of the relevant beliefs about violence. The third study $(N=243)$ shows the definitive design of the Cuestionario de Investigación de la Conducta Violenta Exogrual (CINCOVE) and presents the regression model conducted to explain the intention of carrying out violent behaviour. Results. The final model obtained explains $61.5 \%$ of the variance of the dependent variable "Intention" and identifies the main beliefs related to the intention to carry out violent behaviour on the side of youth belonging to groups. Discussion. The results may contribute to the foundation of the design, implementation and evaluation of interventions that have as main objective the prevention or treatment of the problem.

Key words: Group juvenile violence; health; attitudes: behavioural intention; regression analysis; prevention.

El criterio utilizado para definir estos grupos orientará las estrategias adoptadas para afrontar el problema; en función de dicho criterio las investigaciones y los programas de prevención o intervención derivados de éstas serán más o menos eficaces (Rosenblat, 2007). Sin embargo, son muchas las definiciones de banda o grupo violento juvenil.

Así, por ejemplo, en el Informe Mundial sobre la Violencia y la Salud, se define la violencia como "el uso intencional de la agresión física o de amenazas de agresión contra uno mismo o contra otra persona, grupo o comunidad que tenga como consecuencia principal una alta probabilidad producir lesiones, la muerte, mal desarrollo o privación" (Krug et al. 2003, p.5). La red europea Eurogang (dedicada al estudio comparado de las bandas o pandillas juveniles a nivel europeo) define el término bandas como: "un grupo juvenil duradero, con orientación hacia la calle y otros espacios públicos y con una identidad grupal definida de forma primordial por la participación en actividades delictivas" (Klein, Weerman y Thornberry, 2006, p.418).

En nuestro país, la definición oficial de banda juvenil la recoge la Instrucción 23/2005, de 7 de diciembre, de la Secretaría de Estado de Seguridad desarrollada a partir de las primeras detenciones de los Latin King en España; en ella se define a la banda como un grupo compuesto por jóvenes de entre doce y treinta y dos años, que poseen estructuras de cohesión y disciplina internas, realizan conductas violentas y causan alarma social.

Como podemos observar en los ejemplos anteriores, nos enfrentamos por una parte a definiciones demasiado genéri- 
cas que aconsejan (Chavez, 1999; Moser, 2004) caracterizar el tipo de violencia investigada; en otras ocasiones la propia definición considera otras actividades delictivas además de la violencia propiamente dicha, lo que dificulta el diseño de intervenciones destinadas específicamente a prevenir o tratar este problema.

La investigación sobre el tema en España indica que muchos actos violentos supuestamente individuales, parecen inspirados por normas y hábitos grupales (Martín, Martínez, López, Martín y Martín, 1997). Por ello, investigaciones españolas (Martín, Martínez y Rosa, 2009; Martín et al., 2011; Scandroglio, López y San José, 2008) permiten definir la conducta violenta exogrupal como una agresión física realizada por una o más personas que, en tanto que miembros de un grupo, tratan de provocar intencionalmente daño físico a una o más personas identificadas como miembros de un grupo distinto.

El estudio de actitudes no es la única manera de abordar el análisis de la violencia exogrupal juvenil, pero sí la que más nos interesa: el conocimiento de las actitudes como instrumento de influencia sobre el comportamiento permite explicar conductas individuales y colectivas (Albarracín, Johnson, Zanna y Kumkale, 2005; Eagly y Chaiken, 2007; Maio y Haddock, 2010; Montané, Jarriot y Rodríguez, 2007).

Se ha evidenciado (Díaz-Aguado, 2006) que los sujetos que utilizan una violencia instrumental para lograr sus objetivos la justifican dándole apariencia de legitimidad, por lo que resulta importante establecer las consecuencia (precursoras de las actitudes) que la violencia acarrea para el grupo y sus miembros. La comprensión de la conducta que desarrollan los sujetos pasaría por la indagación de las razones de los mismos para su ejecución o inhibición en un momento dado; y la literatura sobre el tema evidencia (Carrión-María, López-Espín, Gascón-Cánovas y Torres-Cantero, 2012) la relación entre actitudes hacia la violencia y su ejecución efectiva.

Una alternativa psicosocial más compleja e interactiva a la comprensión y modificación de las relaciones entre actitudes y conducta la constituye el modelo teórico. Se han identificado (McGuire, 1989) hasta siete modelos actitudinales; de ellos, uno de los más destacados es el modelo expectativa-valor (Fishbein, 1967; Fishbein y Ajzen, 1975). En este modelo, las creencias se obtienen a partir de los valores esperados referentes a los atributos adscritos al objeto actitudinal. Estos valores tienen dos componentes: la expectativa del atributo es la probabilidad subjetiva de que el objeto actitudinal esté caracterizado por dicho atributo; el valor, por otra parte, es la evaluación subjetiva aplicada al atributo.

Distintos autores (Ajzen, 2001; Eagly y Chaiken, 2007; Eagly, Mladinic y Otto, 1994; Esses, Haddock y Zanna, 1993) han considerado que las personas basan sus juicios actitudinales sobre un conjunto de creencias salientes cuyo número es relativamente restringido: cuando se solicita a los sujetos que manifiesten las creencias sobre un objeto actitudinal, las primeras respuestas elicitadas son las más salientes; si las actitudes son estimadas sobre la base de estas creencias salientes, la correlación con la medida global tiende a ser más alta que cuando se estiman sobre la base de una selección intuitiva impuesta al sujeto por el experimentador.

Igualmente se ha evidenciado (Ajzen y Fishbein, 2005; Doll y Ajzen, 1992; Fazio, 1989; Reagan y Fazio, 1977) que las actitudes basadas en la experiencia directa tienden a ser más fuertes que las formadas a partir de experiencia indirecta y que si tanto la actitud como la conducta están medidas al mismo nivel de generalidad-especificidad, la relación entre ambas aumenta (Jaccard y Blanton, 2005). Ajzen y Fishbein (Ajzen, 1988; Ajzen y Fishbein, 1977) denominaron este presupuesto "principio de compatibilidad o correspondencia" y consideran que cada conducta tiene elementos de acción, meta, contexto y tiempo y que la especificidad o generalidad de la actitud y de la conducta puede ser evaluada en relación a cada uno de estos elementos.

El objeto de estudio de esta investigación es el análisis de la Violencia Juvenil Exogrupal, definida operativamente como la "secuencia conductual realizada por una o más personas, en tanto que miembros de un grupo, dirigida a producir daño físico en uno o más individuos a los que se identifica como pertenecientes a otro grupo distinto". El presente artículo expone el proceso de construcción de un instrumento para medir e identificar las creencias relevantes que los jóvenes integrados en grupos violentos tienen sobre la propia conducta violenta que realizan; una vez identificadas, se utilizarán para explicar el desarrollo de la intención de llevar a cabo este tipo de conductas. El conocimiento de dichas creencias permitiría fundamentar el diseño, la aplicación y la evaluación de intervenciones informativas o educativas que tengan como objetivo principal la prevención o el tratamiento del problema.

\section{Método General}

\section{Participantes}

Todos los participantes (estudios 1, 2 y 3) residían en Madrid en el momento de aplicación de los instrumentos. En el caso de los estudios 1 y 2 fueron contactados en Institutos de Bachillerato, Formación Profesional y Casas de Oficios; el método de captación de participantes para el estudio 3 se detalla en el apartado de Procedimiento. Cada participante fue informado de los objetivos de la investigación y de las garantías de anonimato de su identidad y confidencialidad de sus declaraciones. El protocolo de consentimiento informado fue aprobado por el Comité de Ética de la Universidad Autónoma de Madrid.

Consideramos necesario aclarar los términos "violento" y "no violento" que serán utilizados a continuación. En el primer caso se hace referencia a los jóvenes que declararon haber llevado a cabo la conducta violenta estudiada al menos una vez en el pasado; el término no violento designa a sujetos que declararon no haber realizado nunca la conducta. Los términos utilizados, por tanto, no pueden ni deben ha- 
cerse extensivos al futuro de las personas a las que se les aplica.

\section{Instrumentos}

Para cada estudio se elaboraron cuestionarios específicos que se detallan en su correspondiente apartado. Todos los cuestionarios fueron anónimos y se cumplimentaron de manera individual con ayuda del entrevistador en caso de que fuera preciso.

En los tres instrumentos se formularon los ítems en primera persona para que fueran directos y personales y en todos los casos se determinaba la acción (pegar con mi grupo a una o más personas de otro grupo), la meta (por ejemplo, defender a los amigos del grupo), el contexto (cuando algunos jóvenes se reúnen para salir juntos) y el tiempo (en el próximo mes).

En todos los estudios se incluyó la misma formulación de la conducta analizada.

Conducta. Su formulación fue Pegar, con mi grupo, a una o más personas que pertenecen a otro grupo. Para identificar a los jóvenes con experiencia conductual se incluyó (estudios 1, 2 y 3) la pregunta ¿Has pegado alguna vez, con tu grupo, a una o más personas que pertenecen a otro grupo?; en caso afirmativo, se solicitaba su frecuencia de realización en el último año y en el último mes.

\section{Procedimiento}

En los estudios 1 y 2 se realizó una aplicación masiva de los instrumentos en los centros educativos anteriormente reseñados, teniendo especial cuidado en que la distancia física entre los participantes preservara la privacidad de sus respuestas. Para la aplicación del instrumento definitivo (estudio 3) la captación de participantes se realizó de dos maneras. Por una parte, se contactó con asociaciones de barrio, porteros de discoteca, clubes deportivos y grupos de amigos para localizar a jóvenes que manifestaban realizar la conducta analizada; una vez establecido el contacto con cada uno de ellos, se les aplicó de manera individual el instrumento (captación individualizada). Además, se realizó una aplicación masiva del cuestionario en centros de enseñanza secundaria; de todos los cuestionarios obtenidos, fueron seleccionados únicamente los de aquellos jóvenes que manifestaban pegar con su grupo, siendo desestimado el resto.

\section{Análisis de datos}

El tratamiento y análisis de datos ha sido efectuado mediante el paquete estadístico SPSS 19.0 para Windows.

\section{Primer Estudio: Identificación de las creen- cias salientes sobre violencia juvenil exogrupal}

\section{Método}

Participantes

Para obtener las creencias salientes sobre violencia exogrupal juvenil se utilizó el criterio de similitud con el grupo diana: se conformaron las características de la muestra a las del grupo definido como violentos y descritos en estudios previos en la Comunidad de Madrid: (Martín et al., 2011, 2009) sobre todo varones, con edades comprendidas entre 14 y 22 años.

Participaron 216 jóvenes de entre 14 y 25 años $(M=$ 17.7 ; $D T=1.9)$; el $27.5 \%$ manifestaba realizar la conducta violenta y, de ellos, el porcentaje de varones fue del $52.9 \%$.

\section{Instrumento}

Se elaboró el Cuestionario de Recogida de Creencias sobre Violencia Juvenil Exogrupal que, además de las variables mencionadas en el método general, incluía las siguientes secciones:

Consecuencias. Las dos preguntas formuladas para obtener las consecuencias percibidas sobre la realización de la conducta violenta fueron las siguientes: ¿Qué consecuencias positivas concretas crees que tendría tu acción? y ¿Qué consecuencias negativas concretas crees que tendría tu acción? Ambas iban seguidas de un espacio de 5 líneas para contestar libremente.

\section{Análisis de datos}

Se codificaron las 3 primeras consecuencias positivas y las 3 negativas mencionadas por cada participante. Sobre las respuestas abiertas obtenidas se realizó un análisis categorial que tuvo, en primer lugar, el objetivo de establecer categorías exhaustivas y excluyentes; para ello se utilizaron todas las respuestas, independientemente de que las señalaran jóvenes violentos o no violentos. En segundo lugar, se analizó su frecuencia en la población diana (jóvenes violentos). Las categorías fueron establecidas por un experto (ver Tabla 1) y se consignaron a tres jueces para que verificaran su frecuencia de ocurrencia. El índice de fiabilidad inter-jueces utilizado fue el coeficiente Kappa y el valor alcanzado fue de .8.

\section{Resultados}

La Tabla 1 muestra la proporción de jóvenes que mencionaron cada consecuencia, teniendo en cuenta si manifestaban realizar o no la conducta violenta. Puesto que uno de los objetivos del siguiente estudio era realizar una nueva selección, esta vez en base a criterios estadísticos, no se eliminó ninguna categoría. 
Tabla 1. Consecuencias mencionadas por los encuestados.

\begin{tabular}{lll}
\hline Consecuencias & $\begin{array}{l}\% \\
\text { violentos }\end{array}$ & $\begin{array}{l}\% \\
\text { no violentos }\end{array}$ \\
\hline Miedo a salir a la calle & 65.0 & 5.0 \\
Problemas con familiares/conocidos & 65.0 & 64.0 \\
Crearse enemigos & 37.0 & 16.4 \\
Tener remordimientos & 21.0 & 20.7 \\
Problemas legales (policía, cárcel, etc.) & 19.6 & 40.0 \\
Miedo a ser heridos & 17.4 & 13.0 \\
Miedo a represalias & 13.0 & 30.7 \\
Daños psicológicos a la víctima y a su familia & & 22.0 \\
Aumento de la violencia & & 17.0 \\
Ser humillados & & 28.0 \\
Dar una imagen negativa a los que nos conocen & 13.5 \\
Miedo a herir o matar & 10.9 & 30.7 \\
Sentirse más fuerte & 41.3 & 5.0 \\
Sentirse satisfechos & 26.8 & 8.7 \\
Dar una lección & 21.8 & \\
Ser más respetados & 19.6 & \\
Desahogarme & 17.4 & 10.0 \\
Demostrar superioridad (individual y grupal) & 13.0 & 8.7 \\
Vengarse & 13.0 & 25.0 \\
Sentir el grupo más unido & 8.7 & 25.0 \\
Llamar la atención & 6.5 & 6.3 \\
Defenderse & 6.5 & 3.7 \\
Defender una ideología & 4.3 & 5.0 \\
\hline Nota: Ias casilas vacias incan quen & & \\
\hline
\end{tabular}

Nota: Las casillas vacías indican que la consecuencia no ha sido mencionada en el grupo

Fuente: elaboración propia

Como puede observarse, ambos grupos suelen diferir en sus percepciones sobre las consecuencias de realizar la conducta violenta. Los jóvenes no violentos señalan con bastante frecuencia determinadas consecuencias (daños psicológicos a la víctima y a su familia, aumento de la violencia, ser humillados y dar una imagen negativa) no mencionadas por los jóvenes que sí realizan la conducta violenta; estos, por su parte, identifican con frecuencia ser más respetados y dar una lección. En lo que sí parecen estar de acuerdo ambos grupos es en la idea de que la realización de la conducta violenta les podría ocasionar problemas con familiares o conocidos.

\section{Discusión}

El estudio realizado ha permitido identificar las consecuencias percibidas por los jóvenes acerca de la realización de conductas violentas exogrupales, utilizando para ello el criterio de saliencia. Los resultados obtenidos son consistentes con la idea de que la presencia o ausencia de experiencia directa con la conducta provoca una percepción distinta de las consecuencias derivadas de su realización.

A partir de aquí se diseñó un segundo estudio que permitiera pilotar los resultados obtenidos hasta el momento y seleccionar las consecuencias que mejor relación tuvieran con la intención de realizar la conducta analizada, esta vez en base a criterios estadísticos.

\section{Segundo Estudio: Estudio piloto y Selección estadística de las creencias relevantes sobre violencia juvenil exogrupal}

\author{
Método \\ Participantes
}

Los participantes fueron 250 jóvenes con un rango de edad de entre 14 y 25 años $(M=18.2 ; D T=1.98)$; de ellos, el porcentaje de jóvenes que manifestaron realizar la conducta violenta fue del $22.9 \%$.

\section{Instrumento}

A partir de los resultados obtenidos en el estudio anterior, se elaboró el Cuestionario Piloto sobre Violencia Juvenil Exogrupal. Este instrumento, además de las indicadas en el método general, incluyó las siguientes variables:

Intención. La Intención de realizar la conducta se midió mediante el ítem: Durante el próximo mes tengo la intención de pegar, con mi grupo, a una o más personas que pertenecen a otro grupo, evaluado sobre una escala tipo Likert que va de 1 (Totalmente Falso) a 7 (Totalmente Verdadero).

Creencias. Su medición se realizó mediante dos tipos de ítems: el primero recoge la probabilidad subjetiva de que al realizar la conducta se obtenga una determinada consecuencia (expectativa), mientras que el segundo indaga su valor. La formulación general utilizada para cada una de las consecuencias establecidas en el estudio anterior fue Pegar, con mi grupo, a una o más personas que pertenecen a otro grupo + (consecuencia) evaluada mediante una escala de 1 (Totalmente falso) a 7 (Totalmente verdadero). Para su valoración, se preguntó a los participantes, igualmente mediante una escala de 7 puntos, si para ellos, personalmente, cada consecuencia era 1 (buena) o 7 (mala). Las evaluaciones se intercalaron a las correspondientes consecuencias.

\section{Análisis de datos}

Previamente a la operativización de las creencias se establecieron las formulaciones de las categorías seleccionadas en el estudio anterior. Para ello se llevó a cabo, mediante tres jueces, un análisis de las definiciones y del vocabulario utilizado en las respuestas. Esto es, en cada categoría se seleccionaron las respuestas que eran concretas, directas y fácilmente comprensibles por la mayoría de los adolescentes. El coeficiente Kappa del índice de fiabilidad inter-jueces fue .72 .

Para obtener las creencias se multiplicó cada consecuencia por su valoración; la Tabla 2 recoge las categorías y las correspondientes formulaciones introducidas en el cuestionario. Para analizar las relaciones entre las variables y seleccionar los ítems que serían posteriormente incluidos en el cuestionario definitivo se utilizó el coeficiente de correlación de Pearson. 


\section{Resultados}

De aquéllos jóvenes que manifestaron haber realizado la conducta violenta en alguna ocasión, (el 22.9\% de los encuestados), el 5.6\% manifestó no haberla realizado durante el último año, el 12.4\% señaló pocas veces, el 3.6\% bastantes veces y el 1.2\% indicó muchas veces. En cuanto a la frecuencia de realización de la conducta durante el mes anterior, los participantes señalaron realizarla aproximadamente cada 15 días si bien se observa mucha variabilidad $(M=2.16$; $D T=4.17)$.

En cuanto a la intención de pegar durante el próximo mes, las opiniones aparecen divididas: el 49.1\% de los jóvenes violentos manifiesta ser totalmente $(43.9 \%)$ o bastante $(5.3 \%)$ falso que tengan dicha intención, mientras que el $28.1 \%$ indica algo, el $15.8 \%$ bastante y el $7 \%$ manifiesta ser totalmente verdadero que tengan la intención de realizar dicha conducta en el próximo mes.
Reseñamos a continuación (ver Tabla 2) las correlaciones obtenidas entre las distintas creencias incluidas en el cuestionario y la intención de realizar la conducta violenta en la población de jóvenes violentos.

Los resultados obtenidos indican que la mayoría de las consecuencias seleccionadas en el estudio anterior muestran algún tipo de relación, positiva o negativa, con la intención de realizar la conducta violenta analizada. Merece la pena destacar las elevadas y significativas correlaciones alcanzadas en las formulaciones relacionadas con la idea de respeto, tanto a la propia persona, como a los miembros del endogrupo. Igualmente destacable son las correlaciones obtenidas con las creencias relacionadas con la idea de venganza o dar una lección, siempre relacionadas con el grupo. En el plano personal, se observan tanto consecuencias positivas (sentirse orgulloso, demostrar lealtad, ser popular, por ejemplo) como negativas (crearse enemigos, sentirse mal personalmente, por ejemplo).

Tabla 2. Categorías, formulaciones introducidas en el cuestionario y correlaciones con la intención (solo jóvenes violentos)

Categorías
Prodo a salir a la calle
Crearse enemigos
Tener remordimientos
Problemas legales (policía, cárcel, etc.)
Miedo a ser heridos
Miedo a represalias
Miedo a herir o matar
Sentirse más fuerte
Sentirse satisfechos
Dar una lección
Ser más respetados

Desahogarme

Demostrar superioridad (individual y grupal) Vengarse

Sentir el grupo más unido

Llamar la atención

Defenderse Defender una ideología ${ }^{*} p<.05,{ }^{* *} p<.01, * * * p<.001$ Fuente: elaboración propia

\section{Discusión}

Los resultados obtenidos parecen indicar que la intención de realizar la conducta violenta no parece depender de que las consecuencias percibidas acerca de su realización sean positivas o negativas; la elevada correlación con la in-

\begin{tabular}{ll} 
Formulaciones & $r_{\text {con intención }}$ \\
Me haría tener miedo a salir solo a la calle & .20 \\
Me haría tener problemas con mi familia & $.23^{*}$ \\
Me haría tener problemas con mi novio/a & $.23^{*}$ \\
Haría que la gente de mi barrio me rechazase & .21 \\
Me crearía enemigos & $.42^{* *}$ \\
Me haría sentir mal conmigo mismo & $.35^{* *}$ \\
Me haría tener remordimientos & .25 \\
Me haría tener problemas con la policía & $-.06^{*}$ \\
Haría que fuese a la cárcel & -.11 \\
Podría hacer que me hieran gravemente & .17 \\
Haría que recibiese una paliza & -.22 \\
Podría hacer que hiera gravemente a alguien & $.37^{* *}$ \\
Me haría sentir más fuerte & .13 \\
Me haría sentirme orgulloso de mi mismo & $.38^{* *}$ \\
Haría que diésemos una lección a alguien & $.42^{* *}$ \\
Me haría ser más respetado & $.48^{* * *}$ \\
Haría que respeten a las chicas del grupo & $.26^{*}$ \\
Haría que respeten a los amigos del grupo & $.28^{*}$ \\
Haría que respetasen nuestro territorio & .21 \\
Me haría pasar un buen rato & .24 \\
Me haría humillar a mis enemigos & .23 \\
Haría que me vengase por algo que nos han hecho & $.42^{* *}$ \\
Haría que mi grupo se sintiera más unido & $.34^{*}$ \\
Haría que demostrase lealtad a mis amigos & $.28^{*}$ \\
Haría que me admirasen & .24 \\
Me haría ser popular en mi ambiente & $.45^{* *}$ \\
Me haría ligar más & .25 \\
Me haría sentirme protegido & .08 \\
Haría que respetasen las ideas en las que creemos & $.18^{*}$ \\
\hline
\end{tabular}
Haría que respetasen las ideas en las que creemos

tención de realizar acciones violentas se encuentra en ambos casos. Por otra parte, resulta destacable observar que la intención de realizar la conducta violenta aparece relacionada con que su realización va a traer aparejadas consecuencias que afectan, tanto a la persona considerada individualmente (ser popular, ser más respetado, por ejemplo), como al gru- 
po de referencia (respeto hacia los amigos del grupo, por ejemplo) e, incluso, a la relación entre el joven y su grupo (demostrar lealtad a los amigos, por ejemplo).

Para poner a prueba estas hipótesis y evaluar la importancia de cada una de las variables independientes obtenidas hasta el momento, diseñamos el tercer estudio.

\section{Tercer Estudio: Cuestionario Definitivo sobre Violencia Juvenil Exogrupal}

\section{Método}

\section{Participantes}

La muestra, captada según se indicó en el apartado Procedimiento, se compuso inicialmente de 318 jóvenes que manifestaban realizar la conducta analizada; de ellos, el $48.3 \%$ fueron seleccionados mediante captación individualizada y el $51.7 \%$ en centros educativos. Con la aplicación de diversos filtros, indicados posteriormente (ver apartado Controles de calidad), los participantes definitivos fueron 243 sujetos que declaraban realizar la conducta violenta. La Tabla 3 muestra las características descriptivas de los participantes.

Tabla 3. Características descriptivas de la muestra

\begin{tabular}{|c|c|c|}
\hline Variable & Categoría & Porcentaje \\
\hline \multirow[t]{2}{*}{ Sexo } & Hombre & 78.7 \\
\hline & Mujer & 21.3 \\
\hline \multirow[t]{4}{*}{ Edad (años) } & $13-15$ & 30.1 \\
\hline & $16-18$ & 48.2 \\
\hline & $19-21$ & 14.5 \\
\hline & $>21$ & 7.1 \\
\hline Frecuencia conducta & Ninguna & 34.9 \\
\hline \multirow[t]{9}{*}{ último mes } & 1 & 23.5 \\
\hline & 2 & 6.3 \\
\hline & 3 & 10.3 \\
\hline & 4 & 4.4 \\
\hline & 5 & 7.7 \\
\hline & 6 & 5.9 \\
\hline & 7 & 2.9 \\
\hline & 8 & 2.2 \\
\hline & $>8$ & 1.7 \\
\hline Frecuencia conducta & $1-3$ & 37.3 \\
\hline \multirow[t]{9}{*}{ últimos 12 meses $^{a}$} & $4-6$ & 15.4 \\
\hline & $7-9$ & 3.5 \\
\hline & $10-12$ & 7.1 \\
\hline & $13-15$ & 6.9 \\
\hline & $16-18$ & 0.8 \\
\hline & $19-21$ & 5.0 \\
\hline & $22-24$ & 2.7 \\
\hline & $25-48$ & 11.2 \\
\hline & $>48$ & 4.2 \\
\hline
\end{tabular}

\section{a Número de veces}

Fuente: elaboración propia

El rango de edad de los encuestados osciló entre 13 y 28 años. La mayoría de los participantes $(78.7 \%)$ fueron varones, con una media de edad de 17.26 años $(D T=2.91)$, mientras que la de las mujeres no alcanzó los 17 años $(D T=$ 1.96).

\section{Instrumento}

A partir de los resultados obtenidos en el estudio anterior se elaboró el Cuestionario de Investigación sobre Violencia Juvenil Exogrupal, que incluía las siguientes variables:

Intención. Se efectuaron dos medidas temporales de la Intención (en el próximo mes y en los próximos doce meses) para que los cálculos del encuestado fueran concretos y realistas, y por cada medida se incluyeron dos formulaciones similares en diferentes lugares del cuestionario con el objetivo de servir de control de calidad, según se indica posteriormente. También se preguntó a los participantes si su opinión respecto a la conducta había cambiado en el último año y en el último mes. En ambas se decidió emplear la fórmula "en los últimos doce meses" en lugar de "en el último año" para que los jóvenes no contaran únicamente a partir del mes de enero. Las formulaciones finales fueron las siguientes:

- Durante el próximo mes tengo la intención de pegar con mi grupo, a una o más personas que pertenecen a otro grupo: 1 (Falso) a 7 (Verdadero).

- Durante el próximo mes yo pegaré con mi grupo, a una o más personas que pertenecen a otro grupo: 1 (Falso) a 7 (Verdadero).

- Durante los próximos doce meses tengo la intención de pegar con mi grupo, a una o más personas que pertenecen a otro grupo: 1 (Falso) a 7 (Verdadero).

- Durante los próximos doce meses yo pegaré con mi grupo, a una o más personas que pertenecen a otro grupo: 1 (Falso) a 7 (Verdadero).

- ¿Ha cambiado en el último mes tu opinión sobre la violencia realizada por grupos de jóvenes? 1 (Sí, estoy más en contra) a 7 (Si, estoy más a favor).

- ¿Ha cambiado en los últimos 12 mese tu opinión sobre la violencia realizada por grupos de jóvenes?: 1 (Sí, estoy más en contra) a 7 (Sí, estoy más a favor).

Creencias. Partiendo del estudio anterior, las modificaciones introducidas en la formulación de las consecuencias pretendían mejorar la comprensión de la frase, sin afectar a la homogeneidad de su estructura. Por ello, se intentó conyugar al condicional los auxiliares y no los verbos (formula base: me haría + verbo en infinitivo). En segundo lugar, se procuró que el significado de expectativa fuera preservado evitando el uso de verbos que pudieran dar la idea de consecuencia-medio más que de consecuencia-fin. Sólo en un caso, hacer que me hiriesen, se utilizó el auxiliar podría para evitar una formulación demasiado excesiva; además, el adjetivo gravemente se consideró superfluo o falsamente definitorio y se decidió eliminarlo. En tercer lugar, como el cuestionario iba dirigido a jóvenes violentos, se eligió la formulación de las frases en negativo: por ejemplo, me evitaría tener miedo (más 
probable para los violentos) sustituye la antigua formulación me haría tener miedo (más probable para los no violentos), y evitar que otros me peguen sustituye a recibir una paliza. Finalmente, para conseguir mayor especificidad y la experiencia personal, se sustituyó la formulación defender las ideas en las que creemos por una más personal y autodirigida, las ideas en las que creo, para que el joven se centrara en su conducta y no en la del grupo.

Más allá de estos criterios generales, basándonos en las correlaciones entre las distintas consecuencias, se buscaron formulaciones únicas para aquellos grupos de consecuencias que parecerían pertenecer a una misma categoría. Así, en lugar de me haría tener problemas con mi familia se utilizó me haría tener problemas con las personas que aprecio, abarcando así todos los grupos de referencia importantes para los participantes. En este mismo sentido, defender a los amigos/las chicas del grupo y demostrar lealtad a los amigos fueron unidas en me haría defender a las personas que aprecio. Asimismo, me permitiría castigar a quien se $l o$ merece recoge tanto la idea de dar una lección como de vengarse. El ítem ser respetado es el que mejor relaciona con la Intención y el que mejor recoge la influencia de los ítems ser más temido y ser admirado. En el caso de ir a la cárcel se encontró correlación con tener problemas con la policía pero no con la Intención, así que se decidió eliminarla. Finalmente, en algunos casos se optó por una formulación más amplia que permitiera al sujeto referirse a una categoría de procesos similares: desahogarme en vez de pasar un buen rato, que permite abarcar elementos de activación fisiológica y no sólo de diversión.

Se reformularon dos consecuencias: evitaría que me tomen por un cobarde en vez de ser rechazado, que no correlacionó significativamente con la Intención; me haría sentirme apoyado por mi grupo en vez de que el grupo se sienta más unido, al considerarse más general y dirigida a los elementos afectivos del grupo. Se eliminaron, al no correlacionar con la Intención ni con otras consecuencias: humillar a los enemigos, sentirme más fuerte, recibir una paliza y defender el territorio. La consecuencia ligar más fue eliminada, pues no correlacionaba con la Intención. Así, el número total se redujo a diecisiete; la Tabla 4 muestra su formulación definitiva en el cuestionario.

\section{Análisis de datos}

Se realizaron análisis descriptivos, ofreciéndose índices de tendencia central y desviación, y el nivel de significación obtenido en la prueba $t$ de Student para una muestra, realizada para facilitar la interpretación de datos. El estudio de potenciales relaciones entre variables y su reducción se abordó mediante análisis factorial de componentes principales $(A F C P)$. El criterio de determinación del número de factores a extraer fue el de raíz latente, considerándose únicamente los factores cuyo autovalor fuera mayor que 1 . Los criterios para incluir un ítem en el factor fueron: que los ítemes saturaran por encima de .40 en el factor y que si alguno de los ítemes saturaba en más de un factor, se incluiría en el que la saturación fuera mayor.
Para examinar la contribución de cada variable independiente se realizó un análisis de regresión múltiple ( $A R M)$ utilizando la estimación por etapas (stepwise) introduciendo (Ajzen y Fishbein, 2008; Evans, 1991) tanto las variables por separado (consecuencias y su valoración) como su producto (creencias).

\section{Controles de calidad}

Se eliminaron los cuestionarios en los que el sujeto eludía la respuesta a un número elevado de preguntas (aproximadamente 1 de cada 3) y aquellos en los que la respuesta parecía seguir un patrón (por ejemplo, a modo de dibujo). Además, la inclusión de la doble formulación de la variable intención en distintos lugares del cuestionario permitió fijar un margen de seguridad de la respuesta; para ello, se compararon las respuestas otorgadas por cada sujeto en estos ítems y fueron eliminados aquellos sujetos cuya respuesta a la doble formulación del ítem correspondiente superaba un límite de seguridad que fue establecido en 2 desviaciones estándar. Con estos criterios, la muestra se redujo de 318 a 243 participantes.

\section{Resultados}

Los resultados indican (ver Tabla 4) que la opinión sobre la violencia juvenil parece haberse modificado levemente hacia posiciones menos favorables. En cuanto a la intención de realizar la conducta en el plazo de un mes o de un año, los resultados no muestran importantes diferencias; mayoritariamente, los sujetos manifiestan una intención intermedia, aunque con tendencia en dos casos hacia puntuaciones medias significativamente más bajas que la media de la escala.

En cuanto a las consecuencias manifestadas sobre la violencia, todas excepto dos, las referidas a problemas con las personas que aprecian y con remordimientos (cuyas puntuaciones medias no difieren del término medio de la escala y no pueden, por tanto, se interpretadas en uno u otro sentido), se orientan hacia el polo verdadero. Otro resultado relevante es la mayoritaria percepción de que la violencia exogrupal provoca no sólo efectos sancionados socialmente de modo negativo (problemas con la policía, crearse enemigos, etc.), sino otros con denotaciones culturalmente positivas (proteger a las personas que aprecian, castigar a quien se lo merece, etc.). En el caso de las valoraciones de las consecuencias, la prueba $t$ realizada indica que todas obtienen una puntuación media que difiere significativamente del centro de la escala, es decir, en la totalidad de los ítems los sujetos valoran las consecuencias como buenas o malas.

La importancia del entorno afectivo del sujeto se muestra en la tendencia a extremar las valoraciones de las consecuencias, tanto en sentido positivo (proteger a las personas que aprecio) como negativo (tener problemas con la gente que aprecio). Otro aspecto destacado es la valoración positiva de las consecuencias asociadas al bienestar personal, tanto físico (evitar que me peguen) como psíquico (sentirse protegido, apoyado por el grupo). 
Tabla 4. Estadísticos descriptivos de los ítems del instrumento.

\begin{tabular}{|c|c|c|c|}
\hline Ítems & M & $D T$ & $t$ \\
\hline ¿Ha cambiado en los últimos 12 meses tu opinión sobre la violencia realizada por grupos de jóvenes?a & 3.82 & 1.37 & $* * *$ \\
\hline ¿Ha cambiado en el último mes tu opinión sobre la violencia realizada por grupos de jóvenes?a & 3.51 & 1.23 & $* * *$ \\
\hline Durante el próximo mes tengo la intención de pegar con mi grupo a una o más personas que pertenecen a otro grupo & 3.73 & 2.25 & $*$ \\
\hline Durante el próximo mes yo pegaré con mi grupo a una o más personas que pertenecen a otro grupo ${ }^{b}$ & 3.94 & 2.32 & \\
\hline \multicolumn{4}{|l|}{ Durante los próximos 12 meses tengo la intención de pegar con mi grupo, a una o más personas que pertenecen a otro } \\
\hline grupo $^{\mathrm{b}} \mathrm{H}$ & 3.53 & 2.09 & $* * *$ \\
\hline Durante los próximos 12 meses yo pegaré con mi grupo a una o más personas que pertenecen a otro grupo ${ }^{b}$ & 3.96 & 2.00 & \\
\hline \multicolumn{4}{|l|}{ Consecuencias: Pegar con mi grupo a una o más personas que pertenecen a otro grupo ${ }^{b}$. } \\
\hline ... Me permitiría proteger a las personas que aprecio & 5.69 & 1.79 & $* * *$ \\
\hline ... Me haría tener problemas con la policía & 5.36 & 1.63 & $* * *$ \\
\hline ... Me permitiría castigar a quien se lo merece & 5.28 & 1.87 & $* * *$ \\
\hline ... Me haría sentirme apoyado por mi grupo & 5.14 & 1.91 & $* * *$ \\
\hline ... Me crearía enemigos & 5.12 & 2.12 & $* * *$ \\
\hline ... Me haría herir gravemente a alguien & 5.08 & 2.15 & $* * *$ \\
\hline ... Haría que me respetasen & 5.08 & 1.89 & $* * *$ \\
\hline ... Evitaría que otros me peguen a mí & 5.05 & 2.19 & *** \\
\hline ... Me haría sentirme protegido & 5.03 & 1.98 & $* * *$ \\
\hline ... Me permitiría defender las ideas que creo & 5.03 & 2.12 & $* * *$ \\
\hline ... Podría hacer que me hiriesen & 4.97 & 2.16 & $* * *$ \\
\hline ... Me evitaría tener miedo & 4.56 & 2.31 & $* * *$ \\
\hline ... Me haría ser popular en mi ambiente & 4.16 & 1.98 & $* * *$ \\
\hline ... Evitaría que me tomen por un cobarde & 3.93 & 2.19 & $* *$ \\
\hline ... Me haría desahogarme & 3.83 & 2.20 & $*$ \\
\hline ... Me haría tener problemas con la gente que precio & 3.47 & 2.36 & \\
\hline ... Me haría tener remordimientos & 3.36 & 2.28 & \\
\hline \multicolumn{4}{|l|}{ Valoración: Que yo.......es ${ }^{c}$} \\
\hline ... Proteja a las personas que aprecio & 6.36 & 1.35 & $* * *$ \\
\hline ... Tenga problemas con la policía & 2.03 & 1.51 & $* * *$ \\
\hline ... Castigue a quien se lo merece & 5.06 & 1.87 & $* * *$ \\
\hline ... Me sienta apoyado por mi grupo & 6.04 & 1.49 & $* * *$ \\
\hline ... Me cree enemigos & 2.33 & 1.50 & $* * *$ \\
\hline ... Hiera gravemente a alguien & 2.28 & 1.79 & *** \\
\hline ... Sea respetado & 5.58 & 1.54 & $* * *$ \\
\hline ... Evite que otros me peguen a mí & 6.10 & 1.59 & $* * *$ \\
\hline ... Me sienta protegido & 6.09 & 1.31 & $* * *$ \\
\hline ... Defienda las ideas en las que creo & 6.07 & 1.49 & $* * *$ \\
\hline ... Resulte herido & 1.86 & 1.32 & $* * *$ \\
\hline ... No tenga miedo & 4.41 & 2.54 & $* *$ \\
\hline ... Sea popular en mi ambiente & 4.66 & 1.65 & $* * *$ \\
\hline ... Evite que me tomen por un cobarde & 4.38 & 1.97 & $* *$ \\
\hline ... Me desahogue & 4.78 & 1.97 & $* * *$ \\
\hline ... Tenga problemas con la gente que aprecio & 1.62 & 1.33 & $* * *$ \\
\hline$\ldots$ Tenga remordimientos & 2.85 & 2.12 & $* * *$ \\
\hline
\end{tabular}

... Tenga remordimientos

$* p<.05, * * p<.01, * * * p<.001$

${ }^{\mathrm{a} E s c a l a}$ 1(Si estoy más en contra) a7(Sí estoy más a favor). bescala 1 (Totalmente Falso) a 7 (Totalmente Verdadero). cEscala 1 (Malo) a 7 (Bueno)

Fuente: elaboración propia

Posteriormente se realizó un AFCP con los 4 ítems incluidos para evaluar la intención de realizar la conducta violenta, que identificó en la solución inicial un único factor con autovalor mayor que 1 , y en el que todos los ítemes saturaban por encima del valor utilizado como criterio (.40). Este factor, que explicaba el 77.045\% de la varianza, fue denominado Intención Conductual, agrupa la intención de realizar la conducta estudiada tanto durante el próximo mes como durante el próximo año, y se utilizó como variable dependiente en el posterior análisis de regresión.

Para identificar y ponderar las variables en función de su capacidad explicativa sobre la intención de realizar la con- ducta permitiendo, a su vez, la interpretación particular de cada variable independiente, se realizó un $A R M$ por pasos sucesivos. Como puede observarse en la Tabla 5, el modelo obtenido identifica un total de 12 variables interesantes para explicar la intención de realizar la conducta violenta analizada. El modelo final obtenido para la variable dependiente (Tabla 5) alcanza una capacidad predictiva de algo más del $61 \%\left(R_{\text {corregida }}=61.5 \%\right)$. En la Tabla 5 se pueden observar las variables introducidas en la ecuación con las respectivas estimaciones de los coeficientes del modelo y significación. 
Tabla 5. Resumen del modelo obtenido para la variable dependiente Intención de realizar la conducta violenta.

\begin{tabular}{|c|c|c|c|c|c|c|c|}
\hline & $R$ & $\begin{array}{c}R^{2} \\
\text { ajustada }\end{array}$ & SE & $\begin{array}{c}\text { Cambio } \\
\text { en } R^{2}\end{array}$ & $\begin{array}{c}\text { Cambio } \\
\text { en } F\end{array}$ & $B$ & IC 95\% \\
\hline (Cte), Valoración Consecuencia Tener problemas con la policía & .602 & .360 & .794 & .363 & $137.228^{* * *}$ & $.377 * * *$ & {$[.261, .493]$} \\
\hline Consecuencia Sentirme apoyado por mi grupo & .661 & .433 & .748 & .074 & $31.749 * * *$ & $.180^{* * *}$ & {$[.087, .273]$} \\
\hline Consecuencia Me haría tener problemas con la gente que aprecio & .694 & .475 & .719 & .044 & $20.221 * * *$ & $-.097 *$ & {$[-.207, .014]$} \\
\hline Creencia Podría hacer que me hiriesen & .718 & .507 & .697 & .034 & $16.626 * * *$ & $-.108^{*}$ & {$[-.221, .004]$} \\
\hline Valoración Consecuencia Me evitaría tener miedo & .732 & .525 & .684 & .020 & $10.315^{* *}$ & $.246 * * *$ & {$[.151, .341]$} \\
\hline Valoración Consecuencia Me haría herir gravemente a alguien & .746 & .546 & 669 & .022 & $11.535^{* *}$ & $.214^{* * *}$ & {$[.124, .304]$} \\
\hline Creencia Me evitaría tener miedo & .758 & .561 & .657 & .017 & $9.507 * *$ & $.192 * * *$ & {$[.098, .287]$} \\
\hline Creencia Me crearía enemigos & .769 & .577 & .646 & .017 & $9.587 * *$ & $-.151 * *$ & {$[-.241,-.062]$} \\
\hline Creencia Me permitiría defender las ideas en las que creo & .780 & .593 & .633 & .017 & $10.141 * *$ & $-.115^{* * *}$ & {$[-.178,-.052]$} \\
\hline Creencia Me haría tener problemas con la policía & .787 & .603 & .625 & .012 & $7.034^{* *}$ & $.163^{* *}$ & {$[.060, .267]$} \\
\hline Consecuencia Me permitiría castigar a quien se lo merece & .792 & .610 & .620 & .008 & $5.189 *$ & $.103^{*}$ & {$[.013, .194]$} \\
\hline
\end{tabular}

$* * * p<.001, * * p<.01, * p<.05$

Nota: $N=243$, IC $=$ Intervalo de Confianza, Durbin-Watson $=1.847$

Fuente: elaboración propia

\section{Discusión}

Los resultados obtenidos muestran un buen ajuste global del modelo. De todas las variables independientes utilizadas en el análisis, se han identificado las más relevantes para explicar el desarrollo de la intención de realizar la conducta violenta analizada. De ellas, destacan la consecuencia tener problemas con la policía y las valoraciones de las consecuencias evitar tener miedo y herir gravemente a alguien; el entorno grupal también contribuye de forma notable en el desarrollo de la intención mediante la consecuencia anticipada por el sujeto de que la realización de la conducta violenta le hará sentirse apoyado por el endogrupo. Por otra parte, las creencias de que la realización de la conducta permite defender las ideas en las que uno cree, que dicha acción implicaría el surgimiento de enemigos y la anticipación de posibles problemas con las personas importantes para el sujeto parecen ser las que inhiben el desarrollo de la intención de realizar la conducta violenta.

\section{Discusión y Conclusiones finales}

Los resultados obtenidos nos ayudan a responder a la pregunta básica inicialmente planteada: ¿qué factores afectan a la intención de realizar conductas violentas por parte de jóvenes integrados en grupos? Para formular una respuesta, se deben considerar dos aspectos: la predicción y la explicación.

En términos de predicción, el modelo de regresión obtenido alcanza un elevado nivel de capacidad predictiva mostrando, además, un buen ajuste. La cantidad de varianza explicada alcanza algo más del $60 \%$ y la tasa de error esperada es baja. En el contexto de investigación, los resultados obtenidos permiten explicar una parte sustantiva de la variabilidad de la intención de los jóvenes de realizar conductas grupales violentas.

En términos de explicación de la violencia exogrupal juvenil, se han identificado las creencias, tanto positivas como negativas, que los jóvenes integrados en grupos poseen acerca de la realización de conductas violentas; expectativas fundamentales en estos jóvenes que están directa y positivamen- te relacionadas con intención de realizar la conducta y otras que parecen inhibir o dificultarla. Esto permite establecer algunas de las razones por las que dichas conductas se producen y que tienen una importancia sustancial en el desarrollo de la intención de los sujetos de llevar a cabo dichas acciones.

Con vistas al desarrollo de programas de prevención primaria y secundaria, los resultados muestran la necesidad de valorar la concepción de la conducta violenta por parte de los jóvenes como algo con lo que obtener "beneficios" (sentir el apoyo del grupo, evitar el miedo, etc.) así como que el hecho de anticipar problemas (crearse enemigos, por ejemplo) está relacionado de manera negativa con la intención de realizar este tipo de conductas. Igualmente, se debería prestar atención a la relación de determinadas consecuencias atribuidas por los jóvenes a la violencia, inicialmente negativas (tener problemas con la policía, la posibilidad de herir a otras personas, por ejemplo) pero que muestran una relación directa y positiva con la intención de llevar a cabo la conducta violenta.

Tal y como muestran los resultados, el mantenimiento de una identidad personal (evitar que les peguen, por ejemplo) y social (sentirse protegidos, apoyados por el grupo) positivas, parecen ser principios básicos de la percepción de los sujetos violentos en relación con la apreciación que realizan de las consecuencias asociadas a su conducta. El hecho diferencial de estos jóvenes respecto a otros sectores poblacionales, es que la fuente de esta identidad se concentra en el grupo violento y en su capacidad personal y grupal para realizar actividades violentas con consecuencias, tanto para ellos mismos como para su grupo.

El desarrollo de la intención de realizar la conducta violenta parece centrarse en unas pocas consecuencias que pueden afectar al sujeto (identidad personal), pero también a las personas y grupos relevantes para él (identidad social). Los jóvenes violentos pueden desarrollar pensamiento "contrafáctico" (estimando las consecuencias de realizar o no realizar el comportamiento) o incluso pensar exclusivamente en las consecuencias que acarrearía no comportarse violentamente, desarrollando el comportamiento agresivo por evita- 
ción activa de los efectos negativos. Es probable que este hecho pueda vincularse con las alternativas comportamentales que un sujeto encuentra a su disposición.

Consideramos que los resultados pueden ser interesantes ya que permiten orientar las intervenciones de manera que

\section{Referencias}

Ajzen, I. (1988). Attitudes, personality, and behavior. Chicago: Dorsey.

Ajzen, I. (2001). Nature and operation of attitudes. Annual Review of Psychology; 52, 27-58.

Ajzen, I. y Fishbein, M. (1977). Attitude-behavior relations: A theoretical analysis and review of empirical research. Psychological Bulletin; 84, 888-918.

Ajzen, I. y Fishbein, M. (2008). Scaling and testing multiplicative combinations in the expectancy-value model of attitudes. Journal of Applied Social Psychology; 38 , 2222-2247.

Carrión-María, M. C.; López-Espín, J. J.; Gascón-Cánovas, J. J. y Torres-Cantero, A. M. (2012). Validez y fiabilidad de un cuestionario abreviado para medir actitud autoritaria en adolescentes. Anales de Psicología; 28(1), 188-195.

Doll, J. y Ajzen, I. (1992). Accessibility and Stability of Predictors in the Theory of Planned Behavior. Journal of Personality and Social Psychology; 63 (5), 754-65.

Eagly, A. y Chaiken, S. (2007). The advantages of an inclusive definition of attitude. Social Cognition, 25, 582-602.

Evans, M. G. (1991). The problem of analyzing multiplicative composites. Interactions revisited. American Psychologist, 46, 6-15.

Fiscalía General del Estado (2011). Memoria 2011. Madrid: Fiscalía General del Estado.

Fishbein, M. y Ajzen, I. (1975). Belief, attitude, intention and behavior. An introduction to theory and research. Reading, MA: Addison-Wesley.

Jaccard, J. y Blanton, H. (2005). The origins and structure of behavior: Conceptualizing behavior in attitude research. En D. Albarracín, B. T. Johnson, y M. P. Zanna (Eds.), Handbook of attitudes and attitude change; pp. 125-171. Mahwah, NJ: Erlbaum.

Klein, M. W, Weerman, F. y Thornberry, T. (2006). Street gang violence in Europe. European Journal of Criminology; 3(4), 413-437. combatan o modifiquen las expectativas de los jóvenes hacia la conducta analizada.

Agradecimientos.- Los autores quieren expresar su agradecimiento a todos los participantes por ofrecernos desinteresadamente su tiempo para la cumplimentación de los instrumentos de medida.

Krug, E. G., Linda, L., James, D., Mercy, A., Zwi, A. B. y Lozano R. (Eds.) (2003). Informe mundial sobre la violencia y la salud. Organización Panamericana de la Salud.

Maio, G. y Haddock, G. (2010). The psychology of attitudes and attitude change. London, UK: SAGE Publications Ltd.

Martín, A, Martínez, J. M., López, J., Martín, M. J. y Martín, J. M. (1998). Comportamientos de riesgo: violencia, prácticas sexuales de riesgo y consumo de drogas ilegales en la juventud. Madrid: Entinema.

Martín, M. J., Martínez, J. M., Medina, J. J., Igual, C., Giménez, A., De la Corte, L., et al. (2011). Origen, evolución e involución de los jóvenes pertenecientes a grupos violentos. Murcia: Fundación Diagrama Intervención Psicosocial.

Martín, M. J., Martínez, J. M. y Rosa A. (2009). Las bandas juveniles violentas de Madrid: su socialización y aculturación. Revista Panamericana de Salud Pública; 26(2), 128-136

McGuire, W. J. (1989). The structure of individual attitude systems. En A. R. Pratkanis, S. J. Breckler y A. R. Greenwald (Eds.), Attitude structure and function. Hillsdale, New Jersey: Lawrence Erlbaum Associates.

Ministerio de Interior (2009). Anuario Estadístico del Ministerio de Interior 2009. Madrid: Ministerio de Interior.

Montero, I. y León, O. (2007). A guide for naming research studies in Psychology. International Journal of Clinical and Health Psychology, 7, 847-862.

Rosenblat, J. (2007). Definición y clasificación de pandillas. Recuperado de http://www.oiji.org.

Scandroglio, B., López, J. S. y San José, M.C. (2008). Gangs, youth groups and deviant behaviors: a psycho-social perspective of analysis and intervention, Electronic Journal of Educational Psychology; 14, 6(1), 65-94.

(Artículo recibido: 30-11-2012; revisión recibida: 3-5-2013; aceptado: 28-7-2013) 